\title{
Diabetes Mellitus During Pregnancy Interferes with the Biological Characteristics of Wharton's Jelly Mesenchymal Stem Cells
}

\author{
Laura Pierdomenico ${ }^{1,3,4}$, Paola Lanuti ${ }^{1,3,4}$, Raskit Lachmann ${ }^{1,3}$, Giovanna Grifone ${ }^{5}$, \\ Eleonora Cianci ${ }^{3,4}$, Letizia Gialò ${ }^{1,3}$, Stephanie Pacella ${ }^{1}$, Mario Romano ${ }^{2,3,4}$, \\ Ester Vitacolonna ${ }^{1,3}$, Sebastiano Miscia ${ }^{*, 1,4,}$ and Marco Marchisio ${ }^{1,2,4}$
}

\author{
${ }^{1}$ Department of Medicine and Aging Science (D.M.S.I.), ${ }^{2}$ Department of Biomedical Science, ${ }^{3}$ Center for Aging Sciences \\ (Ce.S.I.), "Università G.d'Annunzio" Foundation, Chieti, Italy, ${ }^{4}$ StemTeCh Group, Chieti, Italy and Institute of \\ Molecular Genetics, National Research Council (CNR) Chieti, Italy
}

\begin{abstract}
Recent research indicates that the origin of obesity and related metabolic disorders is not only caused by genetic and risk factors in adult life (unbalanced diet, insufficient physical activity) but also may be influenced by the perinatal environment. In addition, studies in animal models suggest that the mesenchymal stem cell commitment into pre-adipocytes can already occur during fetal development and perinatal life. Since the number of pre-adipocytes and mature adipocytes is lower in normal subjects than in obese subjects, changes in the prenatal maturational process may play a role in the pathogenesis of obesity and metabolic-associated diseases. Hyperglycemia during pregnancy is related to an increased risk of obesity, early onset of metabolic syndrome and type 2 diabetes in the offspring. For this reason it would be useful to investigate how the perinatal environment may affect fetal mesenchymal stem cells, especially in deregulated gestational diabetes, where the fetal environment is modified in terms of hormone levels and nutrition. Therefore, we have compared Wharton's jelly mesenchymal stem cells (WJ-MSC) obtained from umbilical cord of both healthy and diabetic mothers, in order to better understand the mechanisms involved in metabolic diseases in offspring of diabetic mothers. Results indicate that WJ-MSC from diabetic mothers display, in contrast to cells from healthy mothers, a higher ability to differentiate towards the adipogenic lineage. This suggests that the diabetic uterine environment may be responsible for a "pre-commitment" that could give rise in the post natal life to an alteration of adipocyte production upon an incorrect diet style, which in turn would produce obesity.
\end{abstract}

Keywords: WJ-MSCs, diabetes, differentiation, pregnancy.

\section{INTRODUCTION}

The strict glycemic control is a fundamental factor for reducing maternal and fetal morbidity and complications related to diabetic pregnancies, in cases of pregestational diabetes and in cases of first recognition and diagnosis during pregnancy (Gestational Diabetes Mellitus, GDM). Pregestational diabetes is a known risk factor for defects of the cardiovascular, central nervous, and musculoskeletal systems, even though very important studies indicate that the risk of malformations increases continuously with increasing maternal glycemia during the first 6-8 weeks of gestation. Among diabetic women with good glycemic control, the prevalence of complications and malformations is similar to that in the general population. Gestational diabetes mellitus (GDM) is defined as any degree of glucose intolerance with onset or first recognition during pregnancy [1]. This condition occurs in 1 to $14 \%$ of all pregnancies depending on population characteristics. Recent evidence indicates a worrisome and significant increase in the prevalence of gestational diabetes related to the increase of maternal obesity [2-5]. In

*Address correspondence to this author at the Department of Medicine and Aging Science, Università "G.d'Annunzio" of Chieti-Pescara, Via dei Vestini 31, 66013 Chieti, Italy; Tel: ++39 08713554571; Fax: ++39 08713554568; E-mail: s.miscia@unich.it healthy pregnant women characteristic changes in maternal metabolism provide nutrients for fetal growth while preserving maternal health [6], when pregnancy is complicated by diabetes, a series of maternal-fetal complications can occur [7,8]: development in a diabetic intrauterine environment results in excess fetal growth [4]. The Pedersen hypothesis suggests that maternal hyperglycemia, which is characteristic in diabetes, stimulates fetal pancreas to produce insulin which causes excessive growth or macrosomia [9]. In any case the direct effect of abnormal maternal carbohydrate metabolism is very difficult to be assessed: GDM reflects a metabolically altered fetal environment associated with perinatal morbidity, high birth weight which is also related to later obesity [10-12]. When hyperglycemia occurs during pregnancy (pre gestational diabetes or GDM), there could be malformations, increased fetal growth, maternal and fetal morbidity and increased risk of developing obesity and type 2 diabetes in life [5]. High maternal glucose concentrations are thought to increase maternal weight gain, resulting in feto-placental overgrowth as well as a higher risk of fetal macrosomia, while low maternal glycaemic diets result in normal maternal weight gain and produces infants with birth weights between the 25th and 50th percentile [13]. Recent studies have shown a relationship between elevated maternal glucose concentrations during gestation and increased birth weight and fetal adiposity $[14,15]$. The above evidences call 
to mind the need of a control of the glucose levels before and during pregnancy in order to prevent maternal and fetal complications and to help reducing maternal and fetal trauma at parturition, as well as the risk of obesity-related adult diseases later in the life. Human umbilical cord Wharton's jelly-derived mesenchymal stem cells (WJ-MSCs), obtained from full term fetal membranes immediately after delivery, possess pluripotent characteristics for high "ex-vivo" expansion potential and ability to differentiate into multiple lineages such as bone, cartilage, fat, muscle, liver, heart and brain cells [16-20]. Unlike the isolation of stem cells from other sources, such as embryos and bone marrow, the isolation of WJ-MSCs is a "non invasive" proceedings and does not have surrounding moral or ethical issues [21,22]. Furthermore, WJ-MSCs have a higher proliferation rate and self-renewal capacity compared to other adult stem cells $[23,24]$. In human studies it has been shown that both, the size increase of existing adipocytes and the differentiation of new mature fat cells from mesenchymal precursor cells are mechanisms in developing obesity [25]. This process, called adipogenesis, consists of two related steps: the determination of human mesenchymal stem cells into preadipocytes and the differentiation of preadipocytes into mature fat cells [26]. A recent study in rodents suggests that the determination of mesenchymal stem cells into preadipocytes might occur in very early stages of development, e.g. perinatal life [27]. Since the number of preadipocytes and mature fat cells has been shown to be different between lean and obese human adult subjects [28], variations in the determination process in early stages of adipose tissue development might be important in the pathogenesis of obesity and type 2 diabetes. For this reason it would be useful to investigate how the perinatal environment may affect fetal mesenchymal stem cells, especially in deregulated gestational diabetes, where the fetal environment is modified in terms of hormone levels and nutrition. Therefore, we have compared Wharton's jelly mesenchymal stem cells (WJ-MSC) obtained from umbilical cord in healthy and diabetic women, in order to better understand the mechanisms involved in metabolic diseases in offspring of diabetic mothers.

\section{MATERIAL AND METHODS}

\section{Subjects}

Five healthy and five diabetic pregnant donors were recruited for this study. The study was performed according to the declaration of Helsinki and subsequent revisions [29]. All participants gave informed consent.

\section{Cell Isolation and Cell Culture}

Cell culture institutional review board approval was obtained for all procedures. With the consent of the parents, fresh human umbilical cords were obtained from full-term births; they were aseptically stored in sterile saline solution and processed within 6 hours from partum to obtain umbilical cord mesenchymal stem cells. After the removal of blood vessels, the abundant extracellular matrix of Wharton's jelly was scraped off by a scalpel, finally cutted and centrifuged at $250 \mathrm{Xg}$ for 5 minutes at RT; pellets were washed with serum-free Dulbecco's modified Eagle's medium (D-MEM) (Lonza). Next, cells were treated with collagenase type IV $\left(2 \mathrm{mg} / \mathrm{ml}\right.$ ) (Sigma) for 16 hours at $37^{\circ} \mathrm{C}$, washed in PBS, and treated with $2.5 \%$ trypsin-EDTA (GIBCO) for 30 minutes at $37{ }^{\circ} \mathrm{C}$ under agitation. Finally, cells were washed in PBS and seeded in complete growth medium (HMSCGM) with growth supplements (all from Lonza), in $5 \% \mathrm{CO}_{2}$ in a $37^{\circ} \mathrm{C}$ incubator. Cells were cultured exchanging the medium every 3-4 days until reaching confluence. Adherent cells were detached with $0.05 \%$ trypsin-EDTA, counted by Trypan Blue exclusion test, and re-seeded at $3000 \mathrm{cells} / \mathrm{cm}^{2}$ to reach the $90 \%$ of confluence after 3-4 population doublings [30].

\section{Osteogenic Differentiation}

Cells were plated at a density of $3 \times 10^{3}$ cells $/ \mathrm{cm}^{2}$ and induced to osteogenic differentiation using aMEM (Sigma) $10 \%$ FBS supplemented with $10 \mathrm{mM}$ beta-glycerophosphate (Sigma), $0.2 \mathrm{mM}$ ascorbic acid (Sigma) and $10^{-8} \mathrm{M}$ dexamethasone (Sigma). The medium was changed every 3-4 days for the whole process of differentiation (3 weeks). Osteogenic differentiation was assessed by detection of alkaline phosphatase reactivity [31] and by Alizarin Red S staining [32].

\section{Adipogenic Differentiation}

Cells were plated at a density of $2 \times 10^{4}$ cells $/ \mathrm{cm}^{2}$ and cultured in DMEM high glucose (HG) (Sigma) $10 \%$ FBS, supplemented with $0.5 \mathrm{mM}$ isobutyl methylxanthine (Sigma), $200 \mu \mathrm{M}$ indomethacin (Sigma), 10 $0^{-6} \mathrm{M}$ dexamethasone (Sigma) and $10 \mu \mathrm{g} / \mathrm{ml}$ insulin (Sigma) (induction medium). The differentiation was achieved by alternating cycles every 2-3 days of induction and maintenance (supplemented with only insulin) medium until the end of the process ( 3 weeks). Cells were finally fixed with a solution containing $10 \%$ formalin and stained with fresh oil red O (Sigma) [31]. Real-Time RT-PCR differentiation assessment was performed at term of two weeks of adipogenic induction.

\section{Morphological Images and Elaboration}

Slides were observed with a microscope i50 (Nikon) and images were acquired with a Cool-SNAPcf digital CCD camera (PhotoMetrics, Huntington Beach, CA). Digital processing and elaboration were performed by MetaMorph software (Molecular Devices).

\section{Real-Time RT-PCR}

Total RNA was extracted using the RNeasy Plus Minikit (QIAGEN Inc., Valencia, CA) following the manufacturer's instructions. Reverse transcription was performed with the M-MLV Reverse Transcriptase (Sigma). Real-Time RT-PCR for peroxisome proliferator activated receptor gamma (PPAR $\gamma$ ) and fatty acid binding protein 4 (FABP4) was carried out with the ABI Prism 7900 Sequence Detection System, using commercially available TaqMan Gene Expression Assays (FABP4, Hs01086177_m1, PPAR $\gamma$, Hs01115513_m1) and the TaqMan universal PCR Master Mix (Applied Biosystems, Foster City, CA, USA) according to standard protocols. Beta-2 microglobulin (B2M, 
Hs99999907_m1) (Applied Biosystems, Foster City, CA, USA) was used for template normalization and experimental control as calibrator. Results were analyzed using the $2^{-\Delta \Delta \mathrm{Ct}}$ relative quantification method [33].

\section{Antibodies}

Fluorescein isothiocyanate-conjugated anti-CD13 (CD13-FITC), FITC-conjugated anti-CD44 (CD44-FITC), FITC-conjugated anti-CD45 (CD45-FITC), phycoerythrinconjugated anti-CD29 (CD29-PE), FITC-conjugated antiCD105 (CD105-FITC) and FITC-conjugated anti-CD166 (CD166-FITC) were obtained from Ancell (MN, USA); FITC-conjugated anti-CD14 (CD14-FITC) and PE-conjugated anti-CD133 (CD133-PE) were purchased from Miltenyi Biotec (Bergisch Gladbach, Germany); FITCconjugated anti-CD90 (CD90-FITC), PE-conjugated antiCD73 (CD73-PE), PE-Cy5,5-conjugated anti-ESA (ESAPE-Cy5,5), Alexa488-conjugated Sox2 (Sox2-Alexa488), FITC-conjugated anti-SSEA4 (SSEA4-FITC), PE-conjugated anti-CD146 (CD146-PE), PE-conjugated anti-OCT3/4 (OCT3/4-PE), allophycocyanin-conjugated anti-CD117 (CD117-APC), PE-conjugated anti-CD271 (CD271-PE) and
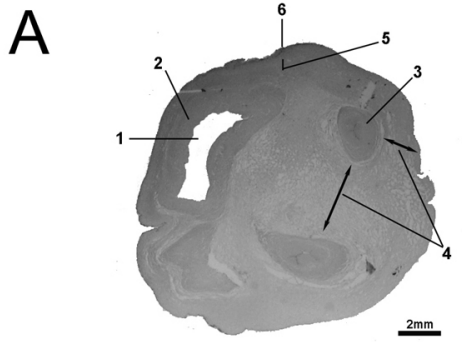

PE-conjugated anti-KDR (KDR-PE) were obtained from Becton Dickinson (BD, San Jose, CA); FITC-conjugated anti-CD144 (CD144-FITC) was obtained from Acris Antibodies (Herford, Germany); PE-conjugated anti-CD34 (CD34-PE) was purchased from Beckman Coulter (Fullerton, CA, USA); TERT primary antibody was obtained from Calbiochem (Dermastadt, Germany) and appropriate secondary FITC-conjugated antibody was obtained from Jackson Immunoresearch Laboratories (West Grove, PA, USA).

\section{Flow Cytometry Staining}

Washing buffer (PBS $0.1 \%$ sodium azide and $0.5 \%$ bovine serum albumine), was used for all washing steps. Samples were stained for surface or intracellular antigens, as previously described [34]. Briefly, $5 \times 10^{5}$ cells/sample were incubated with $100 \mu \mathrm{l}$ of $20 \mathrm{mM}$ ethylene diamin tetraacetic acid (EDTA) at $37^{\circ} \mathrm{C}$ for $10 \mathrm{~min}$. Cells were washed with 3 $\mathrm{ml}$ of washing buffer and centrifuged $\left(4^{\circ} \mathrm{C}, 400 \mathrm{X} \mathrm{g}, 8 \mathrm{~min}\right)$.

Staining of surface antigens. Samples were resuspended in $100 \mu \mathrm{l}$ washing buffer containing the appropriate amount

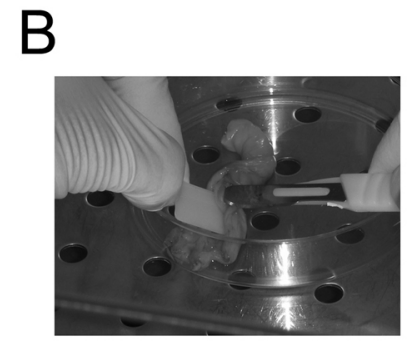

C
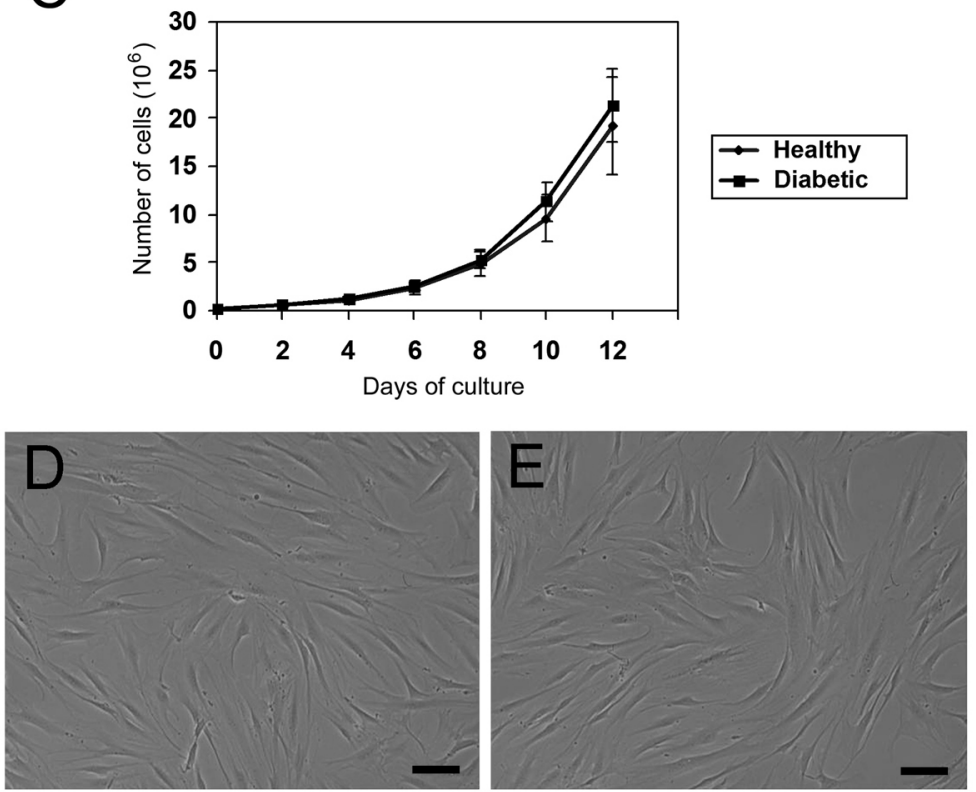

Fig. (1). WJ-MSCs from healthy and diabetic mothers. A) Umbilical Cord Compartment. Digital photo of human umbilical cord tissue. Traverse section through an umbilical cord. Scale bar $=2 \mathrm{~mm}$. 1. Lumen of the vein 2 . Tunica muscolaris of the vein 3 . Tunica muscolaris of the artery 4. Wharton's Jelly 5 . Subamnion 6. Amnion. Wharton's Jelly is the connective tissue between the subamnion and the perivascular region. B) Manual Wharton's Jelly extraction. The image shows the removal of the cord vessels, previous step to scalp Wharton's Jelly allowing the extraction of mesenchymal stem cells. C) Proliferation rate and viability. Proliferation curves of in vitro cultured WJ-MSCs from the umbilical cord of healthy and diabetic mothers were obtained by Trypan blue exclusion test $(\mathrm{n}=5)$. Graphs show means and standard deviations (SD). Phase-contrast images of WJ-MSCs obtained from umbilical cords of healthy (D) and diabetic (E) mothers. Scale bar $=30 \mu \mathrm{m}$. 
of surface antibodies; samples were incubated for $30 \mathrm{~min}$ at $4{ }^{\circ} \mathrm{C}$ in the dark. Cells were washed $(3 \mathrm{ml}$ of washing buffer), centrifuged $\left(4{ }^{\circ} \mathrm{C}, 400 \mathrm{X} \mathrm{g}, 8 \mathrm{~min}\right)$, resuspended with $1 \mathrm{ml} 0.5 \%$ paraformaldehyde, incubated for $5 \mathrm{~min}$ at $\mathrm{RT}$, washed, centrifuged $\left(4{ }^{\circ} \mathrm{C}, 400 \mathrm{X} \mathrm{g}, 8 \mathrm{~min}\right)$ and stored at $4{ }^{\circ} \mathrm{C}$ in the dark until the acquisition.

Staining of intracellular antigens for flow cytometry. Cells were resuspended in $1 \mathrm{ml}$ of FACS Lysing solution (BD), vortexed and incubated at room temperature (RT) in the dark for $10 \mathrm{~min}$. Samples were centrifuged $\left(4{ }^{\circ} \mathrm{C}, 400 \mathrm{X}\right.$ $\mathrm{g}, 8 \mathrm{~min}$ ); $1 \mathrm{ml}$ of Perm 2 (BD) was added to each tube and cells were incubated at RT in the dark for $10 \mathrm{~min}$. Samples were washed and centrifuged $\left(4{ }^{\circ} \mathrm{C}, 400 \mathrm{X} \mathrm{g}, 8 \mathrm{~min}\right)$. Cells were resuspended in $100 \mu \mathrm{l}$ of washing buffer containing the appropriate amount of intracellular antibodies and incubated for $30 \mathrm{~min}$ at $4{ }^{\circ} \mathrm{C}$ in the dark. Cells were centrifuged $\left(4{ }^{\circ} \mathrm{C}\right.$, $400 \mathrm{X} \mathrm{g}, 8 \mathrm{~min}$ ), resuspended with $1 \mathrm{ml} 0.5 \%$ paraformaldehyde, incubated for $5 \mathrm{~min}$ at RT, washed, centrifuged (4 ${ }^{\circ} \mathrm{C}, 400 \mathrm{X} \mathrm{g}, 8 \mathrm{~min}$ ) and stored at $4{ }^{\circ} \mathrm{C}$ in the dark until the acquisition. Cells were analysed on a FACSCalibur flow cytometer (BD), using CellQuest ${ }^{\mathrm{TM}}$ software (BD).

\section{Flow Cytometry Measurement}

Quality control included regular check-up with Rainbow Calibration Particles (BD Biosciences). Debris was excluded from the analysis by gating on morphological parameters; 20,000 non-debris events in the morphological gate were recorded for each sample. To assess non-specific fluorescence we used isotype controls. All antibodies were titrated under assay conditions and optimal photomultiplier voltages (PMT) were established for each channel [35]. Data were analysed using Flow $\mathrm{JO}^{\mathrm{TM}}$ software (TreeStar, Ashland, OR). Mean Fluorescence Intensity Ratio (MFI Ratio) was calculated dividing the MFI of positive events by the MFI of negative events [36].

\section{RESULTS}

\section{Purification and Proliferation Potential of WJ-MSCs}

Fig. (1A) displays the umbilical cord internal organization and the compartment from where WJ-MSCs originate. Fig. (1B) shows the first step of MSC isolation, consisting in
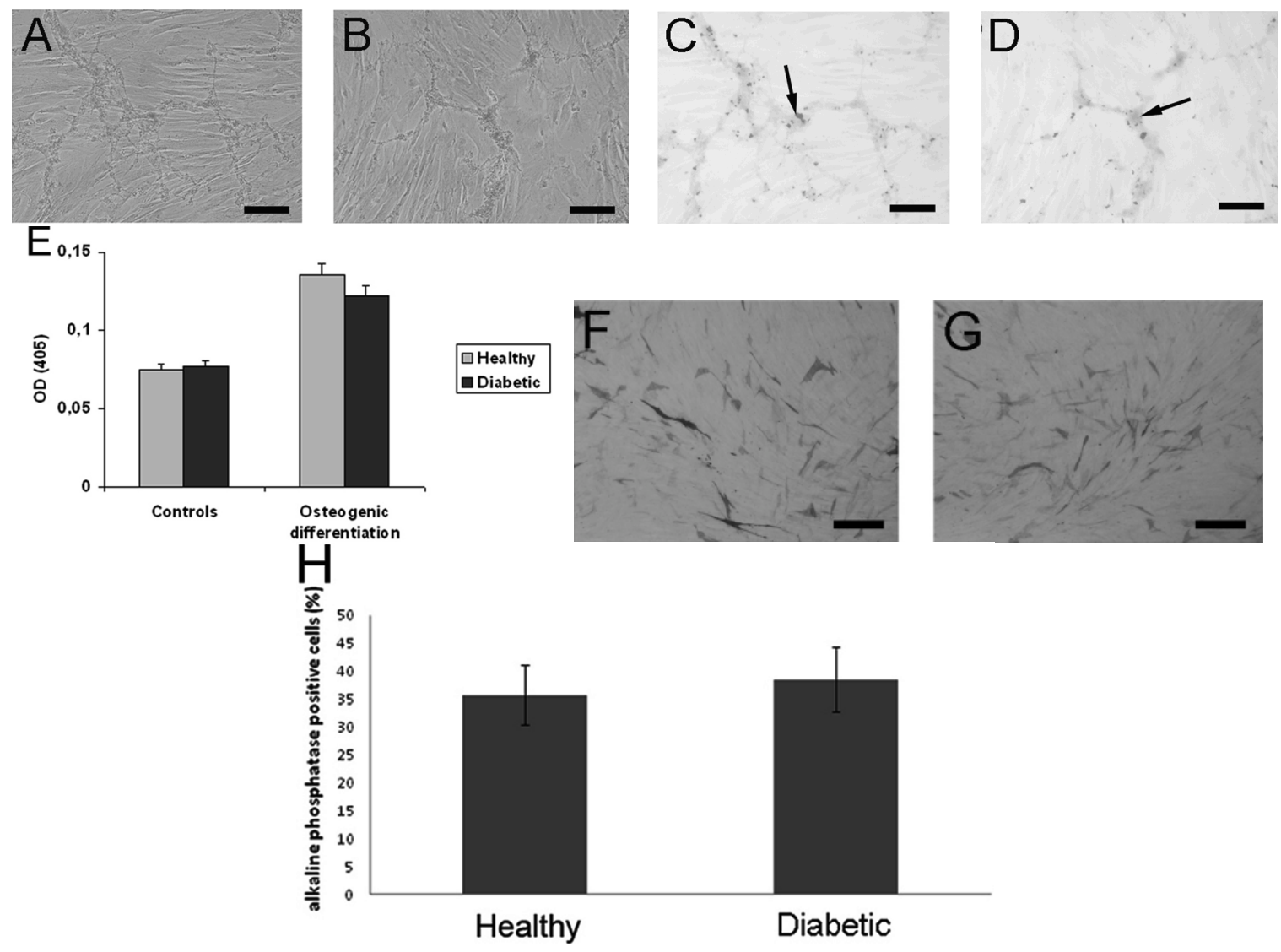

Fig. (2). Osteogenic differentiation ability of WJ-MSCs from healthy and diabetic mothers. Osteogenic differentiation is shown by the Alizarin Red S staining (A-E) and alkaline phosphatase reaction (F-H) in WJ-MSCs obtained from healthy and diabetic mothers. Phasecontrast (A, B) and brightfield (C, D) images obtained with Alizarin Red S staining of WJ-MSCs from healthy (A, C) and diabetic (B, D) mothers. Arrows indicate deposition of extracellular mineralized matrix. Scale bar $=30 \mu \mathrm{m}$. E) Histogram represent the mineralization of WJ-MSC obtained from healthy (gray bars) and diabetic (black bars) mothers by quantification of Alizarin Red S staining via extraction with ammonium hydroxyl. The amount of released dye was measured by a microplate reader at $\lambda 405 \mathrm{~nm}$. The values, are expressed as the mean of optical density units (O.D.), \pm S.D. These data are representative of five biological samples. Brightfield images shown the alkaline phosphatase reaction of WJ-MSCs obtained from healthy (F) and diabetic (G) mothers. Scale bar $=60 \mu$ m. Positivity is represented by the darkening of the cells. H histograms represent the percentage of alkaline phosphatase reaction positive cells obtained by counting the total and positive cells in 10 random fields. Bars represent mean \pm SD of five different biological samples. 
the cord vessel removal, followed by the scalp of Wharton's Jelly and its enzymatic digestion. The collection of WJMSCs umbilical cord-derived from healthy and diabetic mothers showed a comparable success rate (not shown). Different WJ-MSCs derived from umbilical cords of healthy $(\mathrm{n}=5)$ and diabetic $(\mathrm{n}=5)$ mothers were studied for their proliferative ability. No statistically significant differences of the proliferative rates were seen, even if a slight increase of proliferative index was observed in WJ-MSCs from diabetic mothers (Fig. 1C). Primary WJ-MSCs cultures, obtained from umbilical cords of healthy (Fig. 1D) and diabetic (Fig. 1E) mothers showed comparable morphological features.

\section{WJ-MSC Osteogenic Differentiation}

When osteogenic differentiation was induced, the same morphological differentiation were observed in WJ-MSCs phase-contrast images obtained from healthy (Fig. 2A) and diabetic mothers (Fig. 2B) after Alizarin Red S staining. Respect to the phase-contrast images, results obtained by brightfield observation (Fig. (2C) healthy; Fig. (2D) diabetic) broader shows the extracellular mineralized matrix deposition, pointed by arrows. The histogram in Fig. (2E) represents the quantitative evaluation of Alizarin Red $S$ staining by a colorimetric method. There are no significant statistical differences between healthy (gray bars) and diabetic (black bars) WJ-MSC, both in control and osteogenic differentiation conditions. Further confirmation that osteogenic differentiation is comparable in both groups of samples is the frequency of positive cells to the alkaline phosphatase reaction, showed as brightfield images of WJ-MSCs obtained from healthy (Fig. 2F) and diabetic (Fig. 2G) mothers and also showed as percentage of alkaline phosphatase reaction positive cells obtained by counting the total and positive cells (Fig. $2 \mathbf{H}$ ).
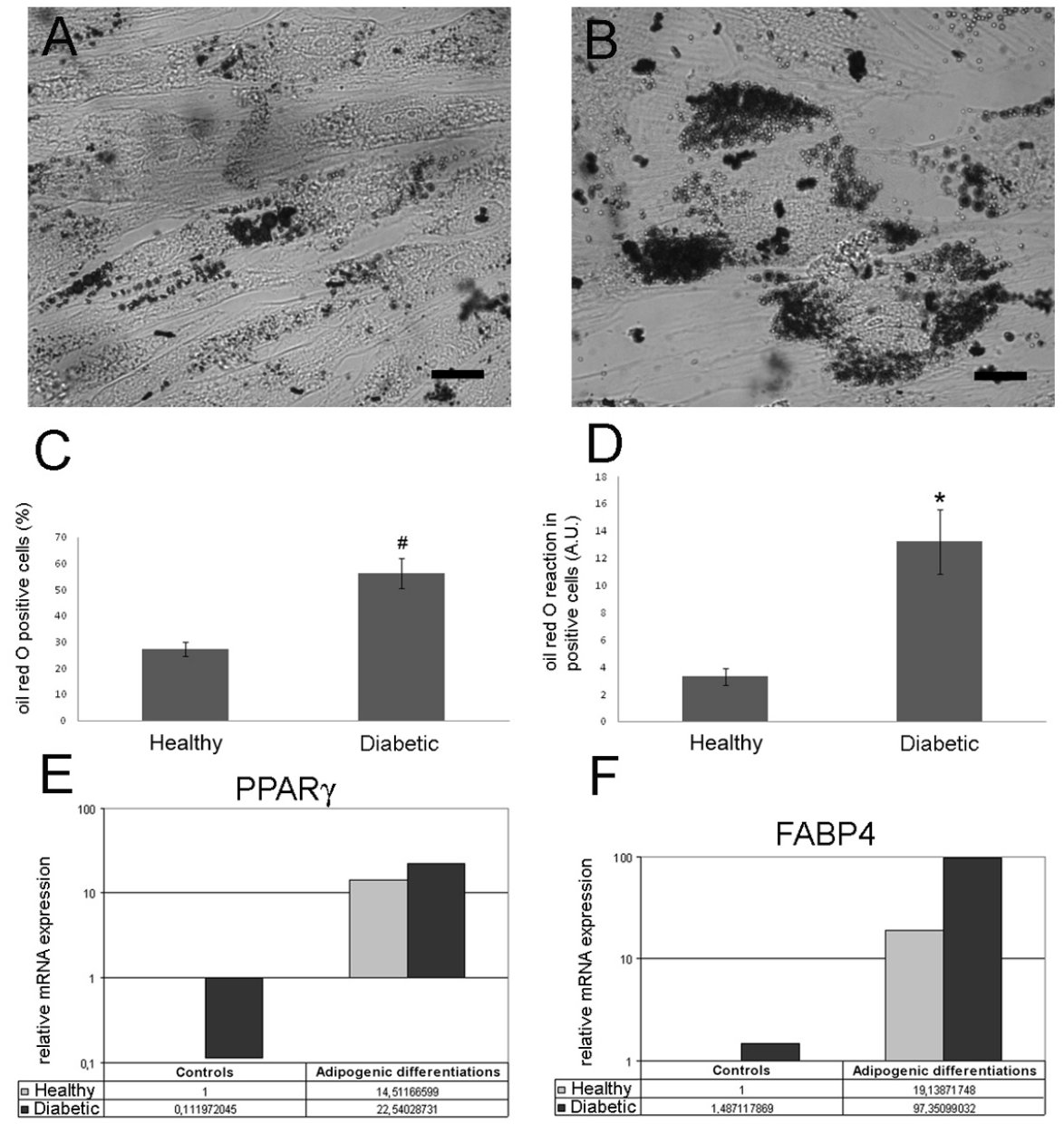

Fig. (3). Adipogenic differentiation ability of WJ-MSCs from healthy and diabetic mothers. Adipogenic differentiation was assessed by accumulation of neutral lipid vacuoles (A-D) and by Real-Time RT-PCR for PPAR $\gamma$ and FABP4 (E, F) Adipogenic differentiation is shown by the accumulation of neutral lipid vacuoles in cultures (oil red O staining) of WJ-MSCs obtained from healthy (A) and diabetic (B) mothers. Scale bar $=15 \mu \mathrm{m}$. C histogram shows the adipogenic differentiation, evaluated in terms of oil red O positive cells (\%) obtained by counting the total and positive cells in 10 random fields. Bars represent mean \pm SD of five different biological samples (\#=p $<0.01)$. D histogram shows the adipogenic differentiation as amount of neutral lipid vacuoles reacting with the oil red O per single positive cells (A.U.) obtained by image analysis (MetaMorph software) of fifty-positive cells of WJ-MSC from healthy and diabetic mothers. Bars represent mean \pm SD of five different biological samples $(*=p<0.001)$. E and $\mathbf{F}$ histograms represent the Real-Time RT-PCR for PPAR $\gamma$ and FABP4 respectively of WJ-MSCs from healthy (gray bars) and diabetic (black bars) mothers. Beta- 2 microglobulin was used for template normalization and healthy control as calibrator. Results were analyzed using the $2^{-\Delta \Delta \mathrm{Ct}}$ relative quantification method. The experiments were performed in technical triplicate. 


\section{WJ-MSC Adipogenic Differentiation}

Adipogenic differentiation of WJ-MSCs obtained from both sources was also induced. The lipid droplets accumulation, after three weeks of induction, was more widespread and abundant in WJ-MSCs obtained from diabetic mothers (Fig. 3B) compared to healthy mothers (Fig. 3A). In detail, Fig. (3C) shows that WJ-MSCs obtained from diabetic mothers presented a statistically significant $(\mathrm{p}<0.01)$ increase of positive cell percentage $(56.3 \pm 4.2)$ to the "oil red O" neutral lipid specific reaction, compared to WJ-MSCs obtained from healthy mothers $(29.1 \pm 1.1)$. To further confirm the different ability of the two WJ-MSC groups to differentiate into adipocytes, the amount of neutral lipid per positive cell was assessed by image analysis (MetaMorph software). WJ-MSCs obtained from diabetic mothers had a high propensity (healthy $3.7 \pm 1.1$ A.U. and diabetic $13.2 \pm$ 2.8 A.U.; $p<0.001)$ to accumulate lipid droplets with respect to WJ-MSCs obtained from healthy mothers (Fig. 3D).

This finding was further confirmed by Real Time RTPCR for PPAR $\gamma$ (Fig. 3E) and FABP4 (Fig. 3F) respectively of WJ-MSCs from healthy (gray bars) and diabetic (black bars) mothers. Adipogenic differentiation leads to a doubling of PPAR $\gamma$ expression (from 14.5116 to $22.54202^{-\Delta \Delta C t}$ ) and a roughly five fold increase of FABP4 expression (from 19.1387 to $97.35092^{-\Delta \Delta C t}$ ) in WJ-MSC from diabetic when compared to healthy mother.

\section{Flow Cytometric Analysis}

Flow cytometric comparison of the two groups was carried out to evaluate differences between the two sources. The phenotypes of the two WJ-MSC groups were comparable, matching with the typical phenotype of MSCs (Fig. 4 and Table 1) [37]. The statistical analysis (Table 1) of MFI ratios showed that both WJ-MSC sources were characterized by a set of antigens expressed at comparable levels (CD13, CD14, CD45, CD144, CD34, CD105, CD133, ESA, KDR, Sox2, CD271, CD146, OCT3/4, CD117). Furthermore Table 1 shows that some other antigens were differently expressed on WJ-MSCs stemming from the two sources. CD44 (healthy $54.6 \pm 3.3$; diabetic $97.9 \pm 4.9 ; \mathrm{p}<0.001$ ), CD29 (healthy $27.4 \pm 1.5$; diabetic $42.3 \pm 2.1 ; \mathrm{p}<0.009$ ), CD73 (healthy $16.1 \pm 1.2$; diabetic $29.5 \pm 1.7 ; \mathrm{p}<0.010$ ), CD166 (healthy $4.4 \pm 0.4$; diabetic $11.8 \pm 0.7 ; \mathrm{p}<0.004$ ), SSEA4 (healthy $3.3 \pm 0.3$; diabetic $8.5 \pm 0.2 ; \mathrm{p}<0.001$ ) and TERT (healthy $278.3 \pm 21.0$; diabetic $513.6 \pm 28.4$; $p<0.002$ ) were higher, whereas CD90 (healthy $69.2 \pm 2.9$; diabetic $54.1 \pm$ $3.3 ; \mathrm{p}<0.008$ ) was lower in WJ-MSCs obtained from diabetic mothers compared to healthy mothers.

\section{CONCLUSION AND DISCUSSION}

Increasing evidence suggests that disturbances in the intrauterine metabolic environment produced by hyperglycemia during pregnancy (pregestational diabetes mellitus and GDM) appear to increase the risk in offspring for obesity and diabetes [11, 12, 38]. There is increasing interest in the hypothesis that cardiovascular and metabolic diseases could be influenced by early life events. Several studies have suggested that exposure to maternal hyperglycemia increases the risk of type 2 diabetes and obesity in later life $[11,12$,
38]. The measures of obesity were significantly correlated with maternal levels of fasting and postprandial blood glucose [39-41]. Catalano et al. published a series of studies comparing the body composition analysis of infants of women with normal glucose tolerance (NGT) and GDM within $48 \mathrm{~h}$ of birth. Although there was no significant difference in birth weight or fat-free mass between the

Table 1. Phenotype and Markers Expression Levels in WJMSC Obtained from Cord of Normal and Diabetic Mothers

\begin{tabular}{|c|c|c|c|c|}
\hline \multirow{2}{*}{ Antigens } & \multirow{2}{*}{ Phenotype } & Healthy (n= 5) & Diabetics (n=5) & \multirow{2}{*}{ P } \\
\cline { 3 - 4 } & & \multicolumn{2}{|c|}{ MFI Ratio \pm S.D. } & \\
\hline \hline CD13 & + & $14.3 \pm 0.3$ & $15.8 \pm 0.6$ & 0.030 \\
\hline CD14 & - & $1.0 \pm 0.1$ & $1.0 \pm 0.1$ & 0.339 \\
\hline CD44 & ++ & $54.6 \pm 3.3$ & $97.9 \pm 4.9$ & 0.001 \\
\hline CD45 & - & $1.0 \pm 0.1$ & $1.0 \pm 0.1$ & 0.500 \\
\hline CD90 & ++ & $69.2 \pm 2.9$ & $54.1 \pm 3.3$ & 0.008 \\
\hline CD144 & $+/-$ & $1.5 \pm 0.1$ & $1.4 \pm 0.1$ & 0.182 \\
\hline CD29 & + & $27.4 \pm 1.5$ & $42.3 \pm 2.1$ & 0.009 \\
\hline CD34 & - & $1.1 \pm 0.1$ & $1.2 \pm 0.1$ & 0.211 \\
\hline CD73 & + & $16.1 \pm 1.2$ & $29.5 \pm 1.7$ & 0.010 \\
\hline CD105 & + & $14.4 \pm 2.4$ & $25.4 \pm 1.7$ & 0.023 \\
\hline CD133 & $+/-$ & $1.4 \pm 0.1$ & $1.7 \pm 0.1$ & 0.126 \\
\hline ESA & - & $1.0 \pm 0.1$ & $1.03 \pm 0.1$ & 0.317 \\
\hline CD166 & + & $4.4 \pm 0.4$ & $11.8 \pm 0.7$ & 0.004 \\
\hline KDR & - & $1.0 \pm 0.1$ & $1.0 \pm 0.2$ & 0.434 \\
\hline Sox2 & ++ & $75.8 \pm 4.4$ & $84.9 \pm 6.7$ & 0.160 \\
\hline SSE44 & + & $3.3 \pm 0.3$ & $8.5 \pm 0.2$ & 0.001 \\
\hline CD271 & - & $1.1 \pm 0.1$ & $1.1 \pm 0.1$ & 0.250 \\
\hline TERT & +++ & $278.3 \pm 21.0$ & $513.6 \pm 28.4$ & 0.002 \\
\hline CD146 & + & $2.6 \pm 0.5$ & $3.8 \pm 0.2$ & 0.068 \\
\hline OCT3/4 & + & $4.0 \pm 0.4$ & $4.7 \pm 0.5$ & 0.137 \\
\hline CD117 & - & $1.2 \pm 0.1$ & $1.3 \pm 0.1$ & 0.107 \\
\hline & + & + & & \\
\hline
\end{tabular}

Numbers in brackets represent the biological samples analyzed; - negative expression; +/- low expression; + moderate expression; ++ positive; +++ high expression; MFI Ratio is the average of five different biological samples \pm standard deviation; $p$ is calculated versus normals MFI Ratio values; Bold values represent MFI Ratio with $\mathrm{p}<$ 0.01 ; Cutoff Ratio positivity $>1.5$.

groups, there was a significant increase in fat mass and percent body fat in the infants of the GDM mothers. There was again a significant increase in fat mass, percent body fat, and skinfold measures in the infants of the GDM mothers compared with the NGT [42]. The HAPO Study has demonstrated a continuous relation between maternal blood glucose and perinatal macrosomia and adiposity [14,15]. Linday et al., recently showed an increased risk of overweight and obesity in offspring of mothers with Type 1 Diabetes [43]. The increase of the prevalence of obesity, diabetes 2 and cardiovascular disease constitutes a "health threat to the individual and it is a major burden for health economy" [38] and several studies have pointed out the possible role of 



Fig. (4). Flow cytometric analyses of surface and intracellular markers. Flow cytometric analysis of WJ-MSC surface and intracellular antigen expression profile: CD13, CD14, CD29, CD34, CD44, CD45, CD73, CD90, CD105, CD117 CD133, CD146, CD166, CD271, ESA, KDR, Sox-2, SSEA4, TERT and OCT3/4. Filled histograms represent cells stained with the expression markers; empty histograms show the respective IgG isotype control. These data are representative of five separate biological samples.

intrauterine hyperglycemia in the pathogenesis of obesity and type 2 diabetes $[38,44]$. Following the above evidences we have decided to study the effect of maternal glycemia on the differentiation of mesenchymal stem cells. A recent 
study in rodents suggests that the determination of mesenchymal stem cells into preadipocytes might occur in very early stages of development, e.g. perinatal life [27]. Since the number of preadipocytes and mature fat cells has been shown to be different between lean and obese human adult subjects [28], variations in the determination process in early stages of adipose tissue development might be important in the pathogenesis of obesity and type 2 diabetes. For this reason it would be useful to investigate how the perinatal environment may affect fetal mesenchymal stem cells, especially in deregulated diabetes during pregnancy, where the fetal environment is modified in terms of hormone levels and nutrition. Therefore, we have compared Wharton's jelly mesenchymal stem cells (WJ-MSC) obtained from umbilical cord in healthy and diabetic women, in order to better understand the mechanisms involved in metabolic diseases in offspring of diabetic mothers. WJ-MSC of healthy and diabetic mothers were morphologically comparable and disclosed similar phenotypes. Although the same markers were expressed in WJ-MSCs obtained from healthy or diabetic mothers, their expression levels differed possibly in relationship with different functional characteristics of the two WJ-MSC groups. We demonstrated lower levels of CD90 in WJ-MSCs from diabetic mothers, which could be associated to a plasticity decrease of these cells [45]. We also showed that WJ-MSCs from diabetic mothers presented a higher adipocyte differentiation efficiency, compared to WJMSCs obtained from healthy mothers, suggesting, therefore, a possible pre-commitment of these cells to the adipogenic lineage. In addition, the up-regulation of CD44, CD29, CD73, CD166, SSEA4 and TERT in WJ-MSCs obtained from diabetic mothers, shown in this study, might be related to the slight increase of proliferative ability of these cells. With regard to the plasticity, WJ-MSCs obtained from diabetic mothers were significantly more oriented to the adipogenic differentiation, as compared to WJ-MSCs obtained from healthy mothers, and as demonstrated by the presence of more evident a higher preadipocyte number, as well as an increased lipid droplet size other than by up regulation of PPAR $\gamma$ and FABP4. The increased capacity of the WJ-MSCs obtained from diabetic mothers to differentiate into adipogenic lineage suggests that the uterine environment induces a series of changes responsible for a "pre-commitment". For this reason, it is conceivable that the uterine environment influences these cells and, following an unbalanced diet in the postnatal life, could result in an abnormal accumulation of adipose tissue. Leading to the development of severe obesity associated with metabolic-related disorders. These data, provide interesting cues that could contribute to explain the pathophysiological mechanisms of obesity origin and associated metabolic disorders.

\section{ACKNOWLEDGEMENTS}

This work was supported by "Carichieti" fondation, Chieti, Italy and by Italian Ministry of Education, University and Research (MIUR): Co-funding research projects of national interest (COFIN) 2007 Grant and FIRB 2010 "accordi di programma".

\section{CONFLICT OF INTEREST}

None declared.

\section{REFERENCES}

[1] American Diabetes Association. Diagnosis and classification of diabetes mellitus (Position Statement). Diabetes Care 2009; 32: S62-S7.

[2] Metzger BE, Coustan DR. Summary and recommendations of the Fourth International Workshop-Conference on Gestational Diabetes Mellitus: the organizing committee. Diabetes Care 1998; 21: B161-B7.

[3] Jovanovic L, Pettitt DJ. Gestational diabetes mellitus. JAMA 2001; 286: $2516-8$.

[4] Dabelea D, Snell-Bergeon JK, Hartsfield CL, Bischoff KJ, Hamman RF, McDuffie RS. Increasing prevalence of gestational diabetes mellitus (GDM) over time and by birth cohort: Kaiser Permanente of Colorado GDM Screening Program. Diabetes Care 2005; 28: 579-84.

[5] Dabelea D. The Predisposition to Obesity and Diabetes in Offspring of Diabetic Mothers. Diabetes care 2008; 30: 169-74.

[6] Freinkel N, Phelps R, Metzger BE. The mother in pregnancies complicated by diabetes, in: Rifkin H, Porte DJ (Eds.), Diabetes Mellitus Theory and Practice, 4th ed., Elsevier, New York, 1990; pp: 634-50.

[7] Gabbe SG. Gestational diabetes mellitus, N Engl J Med 1986; 315 : 1025-30.

[8] Kitzmiller JL. Macrosomia in the infant of diabetic mothers: characteristics, causes, prevention, in: Jovanovic L, Peterson CM, Fuhrmann K (Eds.), Diabetes and Pregnancy: Teratology, Toxicology, and Treatment, Praeger Publishers, New York, 1986; pp: $85-120$.

[9] Pedersen J, Weight and length at birth of infants of diabetic mothers. Acta Endocrinol 1954; 16: 330-42.

[10] Nelson RG, Saad MF, Bennett PH, Knowler WC. Diabetes and obesity in the offspring of Pima Indian women with diabetes during pregnancy. Diabetes Care 1993; 16: 310-4.

[11] Dabelea D, Pettitt DJ. Intrauterine diabetic environment confers risks for type 2 diabetes mellitus and obesity in the offspring, in addition to genetic susceptibility. J Pediatr Endocrinol Metab 2001; 14: 1085-91.

[12] Gillman MW, Rifas-Shiman S, Berkey CS, Field AE, Colditz GA. Maternal gestational diabetes, birth weight, and adolescent obesity. Pediatrics 2003; 111: 221-6.

[13] Clapp JF III. Maternal carbohydrate intake and pregnancy outcome. Proc Nutr Soc 2002; 61: 45-50.

[14] HAPO Study Cooperative Research Group: Metzger BE, Lowe LP, Dyer AR, Trimble ER, Chaovarindr U, Coustan DR, Hadden DR, McCance DR, Hod M, McIntyre HD, Oats JJ, Persson B, Rogers MS, Sacks DA. Hyperglycemia and adverse pregnancy outcomes. N Engl J Med 2008; 358: 1991-2002.

[15] HAPO Study Cooperative Research Group: Hyperglycemia and Adverse Pregnancy Outcome (HAPO) Study: associations with neonatal anthropometrics. Diabetes 2009; 58: 453-9.

[16] Anzalone R, Lo Iacono M, Corrao S, et al. New emerging potentials for human Wharton's jelly mesenchymal stem cells: immunological features and hepatocyte-like differentiative capacity. Stem Cells Dev 2010; 19: 423-38.

[17] Ma L, Feng XY, Cui BL, et al. Human umbilical cord Wharton's Jelly-derived mesenchymal stem cells differentiation into nervelike cells. Chin Med J 2005; 118: 1987-93.

[18] Wang L, Ott L, Seshareddy K, Weiss ML, Detamore MS Musculoskeletal tissue engineering with human umbilical cord mesenchymal stromal cells. Regen Med 2011; 6: 95-109.

[19] Kode JA, Mukherjee S, Joglekar MV, Hardikar AA. Mesenchymal stem cells: immunobiology and role in immunomodulation and tissue regeneration. Cytotherapy 2009; 11: 377-91.

[20] Pereira WC, Khushnooma I, Madkaikar M, Ghosh K. Reproducible methodology for the isolation of mesenchymal stem cells from human umbilical cord and its potential for cardiomyocyte generation. J Tissue Eng Regen Med 2008; 2: 394-9. 
[21] Denker H. Embryonic stem cells: An exciting field for basic research and tissue engineering, but also an ethical dilemma? Cells Tissues Organs 1999; 165: 246-9.

[22] Romano G. Stem cell transplantation therapy: Controversy over ethical issues and clinical relevance. Drug News Perspect 2004; 17: 637-45.

[23] Izadpanah R, Trygg C, Patel B, Kriedt C, Dufour J, Gimble JM, Bunnell BA. Biologic properties of mesenchymal stem cells derived from bone marrow and adipose tissue. J Cell Biochem 2006; 99: 1285-97.

[24] Baksh D, Yao R, Tuan RS. Comparison of proliferative and multilineage differentiation potential of human mesenchymal stem cells derived from umbilical cord and bone marrow. Stem Cells 2007; 25: 1384-92.

[25] Kahn BB, Flier JS. Obesity and insulin resistance. J Clin Invest 2000; 106: 473-81.

[26] Bowers RR, Lane MD. A role for bone morphogenetic protein-4 in adipocyte development. Cell Cycle 2007; 6: 385-9.

[27] Tang W, Zeve D, Suh JM, et al. White fat progenitor cells reside in the adipose vasculature. Science 2008; 322: 583-6.

[28] Tchoukalova Y, Koutsari C, Jensen M. Committed subcutaneous preadipocytes are reduced in human obesity. Diabetologia 2007; 50: 151-7.

[29] World Medical Association General Assembly. World Medical Association Declaration of Helsinki: ethical principles for medical research involving human subjectsjavascript:AL_get(this, 'jour', 'J Int Bioethique.'); 2004; 15: 124-9.

[30] Angelucci S, Marchisio M, Di Giuseppe F, et al. Proteome analysis of human Wharton's jelly cells during in vitro expansion. Proteome Sci 2010; 8: 18.

[31] Pierdomenico L, Bonsi L, Calvitti M, et al. Multipotent mesenchymal stem cells with immunosuppressive activity can be easily isolated from dental pulp. Transplantation 2005; 80: 836-42.

[32] D'Alimonte I, Nargi E, Mastrangelo F, et al. Vascular endothelial grotwh factor enhances in vitro proliferation and osteogenic differentiation of Human dental pulp stem cells. J Biol Regul Homeost Agents 2011; 25: 57-62.

[33] Livak KJ, Schmittgen TD. Analysis of relative gene expression data using real-time quantitative PCR and the $2^{-\Delta \Delta \mathrm{Ct}}$ method. Methods 2001; 25: 402-8.
[34] Lanuti P, Fuhrmann S, Lachmann R, Marchisio M, Miscia S, Kern F. Simultaneous characterization of phospho-proteins and cell cycle in activated T cell subsets. Int J Immunopathol Pharmacol 2009; 22: 689-98.

[35] Perfetto SP, Ambrozak D, Nguyen R, Chattopadhyay P, Roederer M. Quality assurance for polychromatic flow cytometry. Nat Protoc 2006; 1: 1522-30.

[36] Miscia S, Ciccocioppo F, Lanuti P, et al. Abeta(1-42) stimulated T cells express P-PKC-delta and P-PKC-zeta in Alzheimer disease. Neurobiol Aging 2009; 30: 394-406.

[37] Can A, Karahuseyinoglu S. Concise Review: human unbilical cord stroma whit regard to source of fetus-devived stem cells. Stem cells 2007; 25: 2886-95.

[38] Clausen TD, Mathiesen ER, Hansen T, et al. High prevalence of type 2 diabetes and pre-diabetes in adult offspring of women with gestational diabetes mellitus or type 1 diabetes: the role of intrauterine hyperglycemia. Diabetes Care 2008; 31:340-6.

[39] Silverman BL, Rizzo T, Green OC, et al. Long-term prospective evaluation of offspring of diabetic mothers. Diabetes 1991; 40: 121-5.

[40] Pettitt DJ, Bennett PH, Knowler WC, Baird HR, Aleck KA. Gestational diabetes mellitus and impaired glucose tolerance during pregnancy: long-term effects on obesity and glucose tolerance in the offspring. Diabetes 1985; 34: 119-22.

[41] Weiss PA, Scholz HS, Haas J, Tamussino KF, Seissler J, Borkenstein MH. Longterm follow-up of infants of mothers with type 1 diabetes: evidence for hereditary and nonhereditary transmission of diabetes and precursors. Diabetes Care 2000; 23: 905-11.

[42] Catalano PM, Thomas A, Huston-Presley L, Amini SA. Phenotype of infants of mothers with Gestational diabetes. Diabetes Care 2007; 30: S156-60.

[43] Lindsay RS, Nelson SM, Walker JD, et al. Programming of adiposity in offspring of mothers with type 1 diabetes at age 7 years. Diabetes Care 2010; 33: 1080-5.

[44] Gillman MW. Developmental origins of health and disease. N Engl J Med 2005; 353: 1848-50.

[45] Kolf CM, Cho E, Tuan RS. Biology of adult mesenchymal stem cells: regulation of niche, self-renewal and differentiation. Arthritis Res Therapy 2007; 9: 204. 